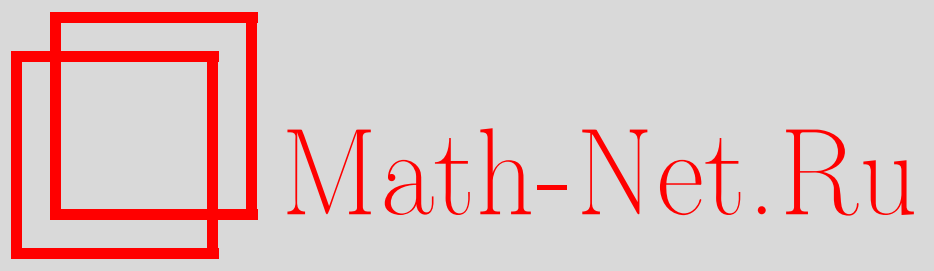

В. И. Ноздрунов, Подход к классификации булевых бент-функций степени нелинейности 3, Дискрет. матем., 2014, том 26, выпуск 4, 59-65

DOI: https://doi.org/10.4213/dm1305

Использование Общероссийского математического портала Math-Net.Ru подразумевает, что вы прочитали и согласны с пользовательским соглашением http: //www . mathnet.ru/rus/agreement

Параметры загрузки:

IP : 3.85 .73 .92

26 апреля 2023 г., $12: 39: 53$

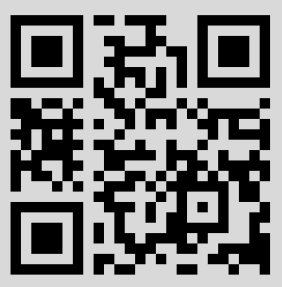




\title{
Подход к классификации булевых бент-функций степени нелинейности 3
}

\author{
() 2014 г. В. И. Ноздрунов*
}

\begin{abstract}
Рассматривается подход к классификации булевых бент-функций степени нелинейности 3 от $n$ переменных. При этом используется аппарат бент-прямоугольников, введенных С. В. Агиевичем. Данный аппарат использовался при классификации булевых бент-функций третьей степени от 8 переменных. Результаты, полученные в данной работе, позволяют строить бент-функции степени нелинейности 3 от произвольного четного количества переменных, с помощью хорошо изученных квадратичных бент-функций.
\end{abstract}

Ключевые слова: бент-функции, бент-прямоугольники, квадратичные формы, аффинные преобразования.

Пусть $V_{n}-n$-мерное векторное пространство над полем $G F(2)$. В работе рассматриваются булевы бент-функции $f: V_{n+2} \rightarrow G F(2)$ степени нелинейности 3 , представленные в виде многочлена Жегалкина. Через $W_{\alpha}^{f}=\sum_{y \in V_{n+2}}(-1)^{f(y)+\langle\alpha, y\rangle}$ обозначим коэффициенты Уолша-Адамара функции $f$; запись $\langle\alpha, y\rangle$ означает скалярное произведение векторов $\alpha$ и $y$. Для удобства занумеруем переменные следующим образом $\left(u, v, x_{1}, \ldots, x_{n}\right)$ и обозначим $\mathbf{x}=\left(x_{1}, \ldots, x_{n}\right)$. Рассмотрим два класса бент-функции от $n+2$ переменных. В первый класс войдут функции $f\left(u, v, x_{1}, \ldots, x_{n}\right)$, у которых в каждый моном третьей степени входит переменная $u$. Во второй класс войдут функции, у которых в каждый моном третьей степени входит хотя бы одна из переменных $u$ или $v$. Таким образом, имеем следующие случаи:

$$
\begin{aligned}
\text { 1) } f\left(u, v, x_{1}, \ldots, x_{n}\right) & =u a(v, \mathbf{x})+c(u, v, \mathbf{x}), \\
\text { 2.1) } f\left(u, v, x_{1}, \ldots, x_{n}\right) & =u a(\mathbf{x})+v b(\mathbf{x})+c(u, v, \mathbf{x}), \\
\text { 2.2) } f\left(u, v, x_{1}, \ldots, x_{n}\right) & =u a(v, \mathbf{x})+v b(\mathbf{x})+c(u, v, \mathbf{x}),
\end{aligned}
$$

где $\operatorname{deg} a(v, \mathbf{x})=\operatorname{deg} a(\mathbf{x})=\operatorname{deg} b(\mathbf{x})=2, \operatorname{deg} c(u, v, \mathbf{x}) \leqslant 2$.

Класс (1) бент-функций рассмотрен в работе [2], поэтому нас интересуют случаи равенств $(2),(3)$. Напомним понятие бент-прямоугольника.

Определение 1. Бент-прямоугольником называется матрица размеров $2^{m} \times 2^{k}$ $(m+k=2 n)$, в которой каждая строка и каждый столбец, умноженный на $2^{(m-k) / 2}$, являются векторами коэффициентов Уолша-Адамара некоторых булевых функций. Множество всех бент-прямоугольников размеров $2^{m} \times 2^{k}$ будем обозначать через $\mathcal{B}_{m \times k}^{\square}$.

* Место работы: Лаборатория ТВП, e-mail: vlad_vin@mail.ru 
Рассмотрим булеву бент-функцию $g$ от $2 n$ переменных. Вектор-строку, полученную транспонированием вектор-столбца табличного задания функции $g$, разобьем на $2^{m}$ подряд идущих подстрок длины $2^{k}$ :

$$
\mathbf{g}=\left(\mathbf{g}_{(\mathbf{1})}, \ldots, \mathbf{g}_{\left(\mathbf{2}^{\mathrm{m}}\right)}\right)
$$

Вектор-строка $\mathbf{g}_{(\mathbf{i})}, i \in \overline{0,2^{m}-1}$, задает некоторую булеву функцию от $k$ переменных, через $\hat{\mathbf{g}}_{(\mathbf{i})}$ обозначим вектор ее коэффициентов Уолша-Адамара. Тогда согласно [1] отображение:

$$
\left(\begin{array}{c}
\mathrm{g}_{(\mathbf{1})} \\
\cdots \\
\mathrm{g}_{\left(2^{\mathrm{m}}\right)}
\end{array}\right) \rightarrow\left(\begin{array}{c}
\hat{\mathrm{g}}_{(\mathbf{1})} \\
\ldots \\
\hat{\mathrm{g}}_{\left(2^{\mathrm{m}}\right)}
\end{array}\right)
$$

задает биекцию между множеством булевых бент-функций от $2 n$ переменных и множеством бент-прямоугольников $\mathcal{B}_{m \times k}^{\square}$. При этой биекции $i$-я строка бент-прямоугольника, соответствующего булевой бент-функции $g\left(x_{1}, \ldots, x_{2 n}\right)$, является строкой коэффициентов Уолша-Адамара следующей функции от $k$ переменных:

$$
g_{\alpha}\left(x_{m+1}, \ldots, x_{2 n}\right)=g\left(\alpha_{1}, \ldots, \alpha_{m}, x_{m+1}, \ldots, x_{2 n}\right),
$$

где $\alpha=\left(\alpha_{1}, \ldots, \alpha_{m}\right)$ вектор из $V_{m}$, а $i=\sum_{j=0}^{m} \alpha_{j} 2^{m-j}$.

Рассмотрим случай равенства $(2)$. Бент-функцию $f\left(u, v, x_{1}, \ldots, x_{n}\right)$ представим следующим образом:

$$
f\left(u, v, x_{1}, \ldots, x_{n}\right)=u\left(a(\mathbf{x})+l_{1}(\mathbf{x})\right)+v\left(b(\mathbf{x})+l_{2}(\mathbf{x})\right)+c^{\prime}(\mathbf{x})+d \cdot u v,
$$

где $\operatorname{deg} a(\mathbf{x})=\operatorname{deg} b(\mathbf{x})=2, \operatorname{deg} c^{\prime}(\mathbf{x}) \leqslant 2$, а $l_{1}(\mathbf{x}), l_{2}(\mathbf{x})-$ линейные функции и $d \in V_{1}$. Построим для функции $f$ бент-прямоугольник размеров $2^{2} \times 2^{n}$. Тогда, как уже было сказано, строками будут являться строки коэффициентов Уолша-Адамара для функций

$$
\begin{aligned}
& f_{1}(\mathbf{x})=f(0,0, \mathbf{x})=c^{\prime}(\mathbf{x}) \\
& f_{2}(\mathbf{x})=f(0,1, \mathbf{x})=b(\mathbf{x})+l_{2}(\mathbf{x})+c^{\prime}(\mathbf{x}) \\
& f_{3}(\mathbf{x})=f(1,0, \mathbf{x})=a(\mathbf{x})+l_{1}(\mathbf{x})+c^{\prime}(\mathbf{x}) \\
& f_{4}(\mathbf{x})=f(1,1, \mathbf{x})=a(\mathbf{x})+b(\mathbf{x})+l_{1}(\mathbf{x})+l_{2}(\mathbf{x})+c^{\prime}(\mathbf{x})+d
\end{aligned}
$$

причем $\operatorname{deg} f_{i}(\mathbf{x})=2$ (этот факт будет доказан ниже). Согласно теореме Диксона (см. [3], [5], [6]) каждая функция $f_{i}(\mathbf{x}), i=1, \ldots, 4$, аффинными преобразованиями приводится к виду

$$
x_{1} \cdot x_{2}+\ldots+x_{2 l-1} \cdot x_{2 l}+c_{1} \cdot x_{2 l+1}+c_{0},
$$

где $2 l-$ ранг квадратичной формы, а $c_{0} \in V_{1}$. Тогда (см. [4]) коэффициенты Уолша-Адамара могут принимать значения только из множества $\left|W_{\alpha}^{f}\right| \in$ $\left\{0,2^{n-\operatorname{rang} f_{i} / 2}\right\}$. Следовательно столбцы бент-прямоугольника могут с точностью до перестановки элементов иметь один из следующих видов:

$$
\left(\begin{array}{l}
0 \\
0 \\
0 \\
0
\end{array}\right),\left(\begin{array}{c} 
\pm 2^{n-\operatorname{rang} f_{i} / 2} \\
0 \\
0 \\
0
\end{array}\right),\left(\begin{array}{c} 
\pm 2^{n-\operatorname{rang} f_{i} / 2} \\
\pm 2^{n-\operatorname{rang} f_{j} / 2} \\
0 \\
0
\end{array}\right),\left(\begin{array}{c} 
\pm 2^{n-\operatorname{rang} f_{i} / 2} \\
\pm 2^{n-\operatorname{rang} f_{j} / 2} \\
\pm 2^{n-\operatorname{rang} f_{t} / 2} \\
0
\end{array}\right),\left(\begin{array}{c} 
\pm 2^{n-\operatorname{rang} f_{i} / 2} \\
\pm 2^{n-\operatorname{rang} f_{j} / 2} \\
\pm 2^{n-\operatorname{rang} f_{t} / 2} \\
\pm 2^{n-\operatorname{rang} f_{k} / 2}
\end{array}\right) .
$$


По определению столбцы данного бент-прямоугольника являются строками коэффициентов Уолша-Адамара для некоторой булевой функции от 2 переменных. Значит, с точностью до перестановки элементов столбцы могут быть только двух видов:

$$
\left(\begin{array}{l} 
\pm 2 \\
\pm 2 \\
\pm 2 \\
\pm 2
\end{array}\right),\left(\begin{array}{c} 
\pm 4 \\
0 \\
0 \\
0
\end{array}\right)
$$

Следовательно, для каждого $i \in\{1, \ldots, 4\}$ имеет место одно из равенств:

$$
2^{-\frac{n}{2}+1} \cdot 2^{n-\operatorname{rang} f_{i} / 2}=4
$$

или

$$
2^{-\frac{n}{2}+1} \cdot 2^{\operatorname{rang} f_{i} / 2}=2
$$

Если выполнено равенство (5), то $\operatorname{rang} f_{i}(\mathbf{x})=n-2$, что соответствует столбцу $\left(\begin{array}{l}4 \\ 0 \\ 0 \\ 0\end{array}\right)$. А если выполнено равенство (6), то $\operatorname{rang} f_{i}(\mathbf{x})=n$, и это соответствует столбцу $\left(\begin{array}{c} \pm 2 \\ \pm 2 \\ \pm 2 \\ \pm 2\end{array}\right)$. Через $E_{i} \subset V_{n}$ обозначим множество векторов $\alpha$ длины $n$, на которых $W_{\alpha}^{f_{i}}$ принимает ненулевые значения.

Методом от противного покажем, что степень каждой функции $f_{i}(\mathbf{x}), i=1, \ldots, 4$, в системе (4) равна 2. Предположим, что ровно одна из функций $f_{i}(\mathbf{x}), i=1, \ldots, 4$, имеет степень, не превосходящую 1. Без ограничения общности будем считать, что $i=1$. Тогда имеем случаи:

$$
\begin{aligned}
& \text { a) } \operatorname{rang} f_{j}(\mathbf{x})=n-2, \quad j \in\{2,3,4\} \text {, } \\
& \text { b) } \operatorname{rang} f_{j}(\mathbf{x})=n, \quad j \in\{2,3,4\} \text {, }
\end{aligned}
$$

В случае $a)$ в бент-прямоугольнике появятся столбцы вида $\left(\begin{array}{l}4 \\ 4 \\ 0 \\ 0\end{array}\right)$ и $\left(\begin{array}{l}0 \\ 0 \\ 0 \\ 0\end{array}\right)$, чего не может быть. В случае $b$ ) появится столбец вида $\left(\begin{array}{c}4 \\ \pm 2 \\ \pm 2 \\ \pm 2\end{array}\right)$, что противоречит определению бент-прямоугольника. Случай, когда две и более функций $f_{i}(\mathbf{x}), i \in\{1, \ldots, 4\}$, имеют степень, не превосходящую 1 , рассматривается аналогично.

Рассмотрим случай равенства (5). Согласно нашим условиям $\operatorname{rang} f_{i}(\mathbf{x})=n-2$, тогда $\left|E_{i}\right|=2^{n-2}$, причем $E_{i} \cap E_{j}=\varnothing$ для любых $i \neq j$, где $i, j \in\{1, \ldots, 4\}$. Покажем, что при определенных условиях с помощью аффинных преобразований вектора переменных $\mathbf{x}=\left(x_{1}, \ldots, x_{n}\right)$ множества $E_{i}$ можно привести к виду

$$
\begin{aligned}
& E_{1}=\left\{(0,0, \mathbf{y}), \mathbf{y} \in V_{n-2}\right\}, \\
& E_{2}=\left\{(0,1, \mathbf{y}), \mathbf{y} \in V_{n-2}\right\}, \\
& E_{3}=\left\{(1,0, \mathbf{y}), \mathbf{y} \in V_{n-2}\right\}, \\
& E_{4}=\left\{(1,1, \mathbf{y}), \mathbf{y} \in V_{n-2}\right\} .
\end{aligned}
$$

Для этого аффинными преобразованиями приведем функцию $f_{1}(\mathbf{x})=c^{\prime}(\mathbf{x})$ к виду $c_{1}+x_{3} x_{4}+\ldots+x_{n-1} x_{n}$, где $c_{1} \in V_{1}$. Соответствующая матрица коэффициентов $C$ 
размеров $n \times n$ будет иметь вид

$$
\left(\begin{array}{cccccccccc}
0 & 0 & \ldots & \ldots & \ldots & \ldots & \ldots & \ldots & 0 & 0 \\
0 & 0 & \ldots & \ldots & \ldots & \ldots & \ldots & \ldots & 0 \\
0 & 0 & 0 & 1 & 0 & 0 & 0 & \ldots & 0 & 0 \\
0 & 0 & 1 & 0 & 0 & 0 & 0 & \ldots & 0 & 0 \\
0 & 0 & 0 & 0 & 0 & 1 & 0 & \ldots & 0 & 0 \\
0 & 0 & 0 & 0 & 1 & 0 & 0 & \ldots & 0 & 0 \\
\ldots & \ldots & \ldots & \ldots & \ldots & \ldots & \ldots & \ldots & \ldots \\
0 & 0 & 0 & 0 & 0 & 0 & 0 & \ldots & 0 & 1 \\
0 & 0 & 0 & 0 & 0 & 0 & 0 & \ldots & 1 & 0
\end{array}\right) .
$$

Выберем матрицы $R_{i}, i \in\{1,2\}$ размеров $n \times n$ вида

$$
\left(\begin{array}{ccccc}
1 & 0 & & \cdots & 0 \\
0 & 1 & \cdots & 0 \\
0 & 0 & & \\
\vdots & \vdots & & R_{i}^{\prime} & \\
0 & 0 & &
\end{array}\right) .
$$

так, чтобы подматрицы $R_{i}^{\prime}$ размеров $(n-2) \times(n-2)$ являлись обратимыми. Зададим две новые матрицы равенствами

$$
\begin{aligned}
& A^{\prime}=R_{1}^{T} C R_{1}, \\
& B^{\prime}=R_{2}^{T} C R_{2},
\end{aligned}
$$

и с их помощью построим матрицы

$$
\begin{aligned}
& A=A^{\prime}-C, \\
& B=B^{\prime}-C .
\end{aligned}
$$

Если соответствующие матрицы коэффициентов квадратичных форм $a(\mathbf{x})$ и $b(\mathbf{x})$ можно построить таким образом, и при этом $A \neq B$, то осуществим переход к системе (7).

Пример 1. Рассмотрим булеву бент-функцию $g$ от 8 переменных:

$$
\begin{aligned}
g\left(u, v, x_{1}, \ldots, x_{6}\right) & =u x_{1} x_{4}+u x_{4} x_{5}+v x_{3} x_{4}+u x_{1}+v x_{2}+v x_{3}+v x_{5}+ \\
& +x_{1} x_{3}+x_{3} x_{5}+x_{1} x_{6}+x_{4} x_{6}+x_{5} x_{6} .
\end{aligned}
$$

Представим функцию в виде

$$
\begin{aligned}
g\left(u, v, x_{1}, \ldots, x_{6}\right) & =u\left(x_{1} x_{4}+x_{4} x_{5}+x_{1}\right)+v\left(x_{3} x_{4}+x_{2}+x_{3}+x_{5}\right)+ \\
& +x_{1} x_{3}+x_{3} x_{5}+x_{1} x_{6}+x_{4} x_{6}+x_{5} x_{6} .
\end{aligned}
$$

Тогда в соответствии с (4) имеем равенства

$$
\begin{aligned}
c^{\prime}(\mathbf{x}) & =x_{1} x_{3}+x_{3} x_{5}+x_{1} x_{6}+x_{4} x_{6}+x_{5} x_{6}, \\
a(\mathbf{x}) & =x_{1} x_{4}+x_{4} x_{5}, \\
b(\mathbf{x}) & =x_{3} x_{4}, \\
l_{1}(\mathbf{x}) & =x_{1}, \\
l_{2}(\mathbf{x}) & =x_{2}+x_{3}+x_{5} .
\end{aligned}
$$


Линейной заменой переменных $\left(x_{1}, \ldots, x_{6}\right)$ с матрицей $Q$ квадратичная форма $c^{\prime}(x)$ приводится к каноническому виду.

$$
Q=\left(\begin{array}{llllll}
1 & 0 & 0 & 1 & 0 & 0 \\
0 & 1 & 1 & 0 & 1 & 0 \\
0 & 0 & 1 & 0 & 0 & 0 \\
0 & 0 & 0 & 1 & 0 & 0 \\
1 & 0 & 0 & 0 & 1 & 0 \\
0 & 0 & 1 & 0 & 0 & 1
\end{array}\right)
$$

После такой замены функция $g$ становится линейно эквивалентной (см. [6]) функции

$$
g^{\prime}\left(u, v, x_{1}, \ldots, x_{6}\right)=u x_{4} x_{5}+v x_{3} x_{6}+u x_{1}+v x_{2}+x_{3} x_{4}+x_{5} x_{6} .
$$

Для этой функции в соответствии с обозначениями (8), (9) матрицы квадратичных форм и матрицы переходов имеют вид

$$
\begin{gathered}
C=\left(\begin{array}{lllllllll}
0 & 0 & 0 & 0 & 0 & 0 & 0 & 0 \\
0 & 0 & 0 & 0 & 0 & 0 & 0 & 0 \\
0 & 0 & 0 & 1 & 0 & 0 & 0 & 0 \\
0 & 0 & 1 & 0 & 0 & 0 & 0 & 0 \\
0 & 0 & 0 & 0 & 0 & 1 & 0 & 0 \\
0 & 0 & 0 & 0 & 1 & 0 & 0 & 0 \\
0 & 0 & 0 & 0 & 0 & 0 & 0 & 1 \\
0 & 0 & 0 & 0 & 0 & 0 & 1 & 0
\end{array}\right), \\
A^{\prime}=\left(\begin{array}{llllll}
0 & 0 & 0 & 0 & 0 & 0 \\
0 & 0 & 0 & 0 & 0 & 0 \\
0 & 0 & 0 & 1 & 0 & 0 \\
0 & 0 & 1 & 0 & 1 & 0 \\
0 & 0 & 0 & 1 & 0 & 1 \\
0 & 0 & 0 & 0 & 1 & 0
\end{array}\right), R_{1}=\left(\begin{array}{lllllll}
1 & 0 & 0 & 0 & 0 & 0 \\
0 & 1 & 0 & 0 & 0 & 0 \\
0 & 0 & 1 & 0 & 0 & 0 \\
0 & 0 & 0 & 1 & 0 & 0 \\
0 & 0 & 1 & 0 & 1 & 0 \\
0 & 0 & 0 & 0 & 0 & 1
\end{array}\right), \\
0
\end{gathered}
$$

Легко заметить, что при этом множества $E_{i}, i \in\{1, \ldots, 4\}$, соответствующие функциям

$$
\begin{aligned}
& g_{1}^{\prime}(\mathbf{x})=g^{\prime}\left(0,0, x_{1} \ldots, x_{6}\right), \\
& g_{2}^{\prime}(\mathbf{x})=g^{\prime}\left(0,1, x_{1} \ldots, x_{6}\right), \\
& g_{3}^{\prime}(\mathbf{x})=g^{\prime}\left(1,0, x_{1} \ldots, x_{6}\right), \\
& g_{4}^{\prime}(\mathbf{x})=g^{\prime}\left(1,1, x_{1} \ldots, x_{6}\right),
\end{aligned}
$$


Итак, функцию $f(u, v, \mathbf{x})$ с помощью афинных преобразований можно представить в виде

$$
\begin{aligned}
f(u, v, \mathbf{x}) & =(u+1)(v+1) f_{1}+(u+1) v f_{2}+u(v+1) f_{3}+u v f_{4}= \\
& =u v\left(f_{1}+f_{2}+f_{3}+f_{4}\right)+u\left(f_{1}+f_{3}\right)+v\left(f_{1}+f_{2}\right)+f_{1} .
\end{aligned}
$$

Используя (7), получаем что функция $f(u, v, \mathbf{x})$ представима в виде

$$
\begin{aligned}
f(u, v, \mathbf{x})=u v\left(f_{1}^{\prime}(\mathbf{y})+f_{2}^{\prime}(\mathbf{y})\right. & \left.+x_{2}+f_{3}^{\prime}(\mathbf{y})+x_{1}+f_{4}^{\prime}(\mathbf{y})+x_{1}+x_{2}\right)+ \\
& +(u+v+1) f_{1}^{\prime}(\mathbf{y})+v f_{2}^{\prime}(\mathbf{y})+v x_{2}+u f_{3}^{\prime}(\mathbf{y})+u x_{1},
\end{aligned}
$$

где $\mathbf{x}=\left(x_{1}, x_{2}, \mathbf{y}\right), \mathbf{y} \in V_{n-2}$, a $f_{i}^{\prime}(\mathbf{y})$-квадратичные бент-функции от $n-2$ переменных, $i \in\{1, \ldots, 4\}$. Напомним, что рассматривается случай равенства (2), где переменные $u$ и $v$ не входят одновременно в мономы степени нелинейности 3: отсюда следует дополнительное условие:

$$
\left[\begin{array}{c}
f_{1}^{\prime}(\mathbf{y})+f_{2}^{\prime}(\mathbf{y})+f_{3}^{\prime}(\mathbf{y})+f_{4}^{\prime}(\mathbf{y})=0 \\
f_{1}^{\prime}(\mathbf{y})+f_{2}^{\prime}(\mathbf{y})+f_{3}^{\prime}(\mathbf{y})+f_{4}^{\prime}(\mathbf{y})=1
\end{array}\right.
$$

которое запишем в виде одного соотношения:

$$
f_{1}^{\prime}(\mathbf{y})+f_{2}^{\prime}(\mathbf{y})+f_{3}^{\prime}(\mathbf{y})+f_{4}^{\prime}(\mathbf{y})=c, c \in V_{1} .
$$

Значит, равенство (10) приобретает вид:

$$
f(u, v, \mathbf{x})=u\left(f_{1}^{\prime}(\mathbf{y})+f_{3}^{\prime}(\mathbf{y})+x_{1}\right)+v\left(f_{1}^{\prime}(\mathbf{y})+f_{2}^{\prime}(\mathbf{y})+x_{2}\right)+f_{1}^{\prime}(\mathbf{y})+c \cdot u v .
$$

Случай (5) рассмотрен, перейдем к случаю, когда выполняется равенство (6). Как уже было сказано, в этом случае $\operatorname{rang} f_{i}(\mathbf{x})=n$, тогда $\left|E_{i}\right|=2^{n}$. Следовательно, функция $f(u, v, \mathbf{x})$ представима в виде

$$
\begin{aligned}
f(u, v, \mathbf{x})=u v\left(f_{1}(\mathbf{x})+f_{2}(\mathbf{x})+f_{3}(\mathbf{x})+f_{4}(\mathbf{x})\right)+ \\
+u\left(f_{1}(\mathbf{x})+f_{3}(\mathbf{x})\right)+v\left(f_{1}(\mathbf{x})+f_{2}(\mathbf{x})\right)+f_{1}(\mathbf{x}) .
\end{aligned}
$$

Так как переменные $u$ и $v$ не входят одновременно в один моном третьей степени, то получаем условие

$$
f_{1}(\mathbf{x})+f_{2}(\mathbf{x})+f_{3}(\mathbf{x})+f_{4}(\mathbf{x})=c, c \in V_{1} .
$$

В итоге получаем равенство

$$
f(u, v, \mathbf{x})=u\left(f_{1}(\mathbf{x})+f_{3}(\mathbf{x})\right)+v\left(f_{1}(\mathbf{x})+f_{2}(\mathbf{x})\right)+f_{1}(\mathbf{x})+c \cdot u v,
$$

где $f_{1}(\mathbf{x})+f_{2}(\mathbf{x})+f_{3}(\mathbf{x})+f_{4}(\mathbf{x})=c, c \in V_{1}$.

Случай равенства (3) рассматривается аналогично, поэтому приведем итоговые результаты.

Утверждение 1. Пусть бент-функиия $f\left(u, v, x_{1}, \ldots, x_{n}\right)$ представима в виде

$$
f\left(u, v, x_{1}, \ldots, x_{n}\right)=u\left(a(\mathbf{x})+l_{1}(\mathbf{x})\right)+v\left(b(\mathbf{x})+l_{2}(\mathbf{x})\right)+c^{\prime}(\mathbf{x})+d \cdot u v .
$$

Тогда, если rang $c^{\prime}(\mathbf{x})=n$, то она эквивалентна функции

$$
f(u, v, \mathbf{x})=u\left(f_{1}(\mathbf{x})+f_{3}(\mathbf{x})\right)+v\left(f_{1}(\mathbf{x})+f_{2}(\mathbf{x})\right)+f_{1}(\mathbf{x})+c \cdot u v ;
$$


если rang $c^{\prime}(\mathbf{x})=n-2$ и матрицы квадратичных форм удовлетворяют условиям (8), (9), то она эквивалентна функции

$$
f(u, v, \mathbf{x})=u\left(f_{1}^{\prime}(\mathbf{y})+f_{3}^{\prime}(\mathbf{y})+x_{1}\right)+v\left(f_{1}^{\prime}(\mathbf{y})+f_{2}^{\prime}(\mathbf{y})+x_{2}\right)+f_{1}^{\prime}(\mathbf{y})+c \cdot u v,
$$

где $f_{i}^{\prime}(\mathbf{y})$ и $f_{i}(\mathbf{x})$ - бент-функции второй степени от $n-2$ и $n$ переменных соответственно.

Утверждение 2. Пусть бент-функиия $f\left(u, v, x_{1}, \ldots, x_{n}\right)$ представима в виде

$$
f\left(u, v, x_{1}, \ldots, x_{n}\right)=u\left(a(\mathbf{x})+v l(\mathbf{x})+l_{1}(\mathbf{x})\right)+v\left(b(\mathbf{x})+l_{2}(\mathbf{x})\right)+c^{\prime}(\mathbf{x})+d \cdot u v .
$$

Тогда, если $\operatorname{rang} c^{\prime}(\mathbf{x})=n$, то она эквивалента функиии

$$
f(u, v, \mathbf{x})=u v \cdot l(\mathbf{x})+u\left(f_{1}(\mathbf{x})+f_{3}(\mathbf{x})\right)+v\left(f_{1}(\mathbf{x})+f_{2}(\mathbf{x})\right)+f_{1}(\mathbf{x})+c \cdot u v ;
$$

если $\operatorname{rang} c^{\prime}(\mathbf{x})=n-2$ и матричь квадратичных форм удовлетворяют условиям (8), (9), то она эквивалентна функиии

$f(u, v, \mathbf{x})=u v \cdot l(\mathbf{y})+u\left(f_{1}^{\prime}(\mathbf{y})+f_{3}^{\prime}(\mathbf{y})+x_{1}+x_{2}\right)+v\left(f_{1}^{\prime}(\mathbf{y})+f_{2}^{\prime}(\mathbf{y})+x_{2}\right)+f_{1}^{\prime}(\mathbf{y})+c \cdot u v$,

где $f_{i}^{\prime}(\mathbf{y})$ и $f_{i}(\mathbf{x})$ - бент-функции второй степени от $n-2$ и $n$ переменных соответственно.

\section{Список литературы}

1. S. V. Agievich, "On the representation of bent functions by bent rectangles", Probabilistic Methods in Discrete Mathematics: Proc. First Intern. Petrozavodsk Conf., 2002, 121-135.

2. S. V. Agievich, "On the affine classification of cubic bent functions", Tr. Inst. Mat., 14:1 (2006), 3-11.

3. Лидл Р.,Нидерейтер Х., Конечные поля., пер. с англ., Мир, Москва, 1989.

4. Логачев О.А., Сальников А.А., Ященко В.В., Булевы функиии в теории кодирования и криптографии., МЦНМО, Москва, 2004.

5. Мак-Вильямс Ф. Дж., Слоан Н. Дж. А., Теория кодов, исправляющих ошибки., пер. с англ., Связь, Москва, 1979.

6. Черемушкин А. В., “Методы аффинной и линейной классификации двоичных функций”, Тр. по дискр. матем., 4 (2001), 273-314. 